\title{
Uso Didáctico de las Tecnologías de la Información y Comunicación en las Universidades: ¿aspiración o expectativa?
}

\author{
Haydee Guillermina Páez \\ hayvelui@gmail.com \\ https://orcid.org/0000-0003-3419-4643 \\ Universidad José Antonio Páez (UJAP) \\ San Diego, Venezuela \\ Nolberto Goncalves Rodríguez \\ nolbertogoncalves@ gmail.com \\ https://orcid.org/0000-0003-4140-8419 \\ Universidad de Carabobo (UC) \\ Bárbula, Venezuela \\ Evelyn Cristina Arreaza Páez \\ evelynarreaza.us@gmail.com \\ https://orcid.org/0000-0002-1757-2545 \\ Harleyville-Ridgeville Middle School \\ South Carolina, Estados Unidos de Norteamérica
}

Recibido: 26/03/2020 Aceptado: 25/05/2020

\begin{abstract}
Resumen
El ser humano siempre ha puesto su curiosidad e ingenio para mejorar su calidad de vida, siendo la comunicación una de las áreas más destacadas debido a su notable rol socializador. La convergencia de los avances en electrónica, informática y comunicaciones transmutó en la telemática y en la creación de la Internet, que ha interconectado al mundo y todos los ámbitos del acontecer social. La educación, proceso netamente social, ha sido impactada por el advenimiento de la Internet desde la década de los noventa, modificando no sólo el tipo de recursos utilizados en el proceso didáctico de facilitación y adquisición de aprendizajes, por sus dos actores protagónicos: docentes y estudiantes, respectivamente; sino también, la cultura de las instituciones educativas, las cuales como en el caso Venezolano, por imperio de la ley, deben incorporar en su misión y planes de desarrollo organizacional, las Tecnologías de la Información y Comunicación (TIC). Transcurridas dos décadas de haber sido establecido como prioridad el uso de la internet en las instituciones educativas, ergo en las Universidades Venezolanas, en el presente artículo se analizan, bajo la visión de los mundos Popperianos (Popper, 1986), dos instituciones universitarias, una pública y otra privada; para determinar si el uso didáctico de las TIC responde a una expectativa o a una aspiración y si ésta, es personal o institucional. Se evidenció una insatisfacción de la expectativa legal decantada en una aspiración institucional, dada la existencia de una generalizada predisposición negativa de docentes y estudiantes hacia el uso de las TIC como recurso para mediar procesos didácticos en la sociedad actual, lo cual resalta el importante papel del componente afectivo personal, en este caso la afectividad digital (Goncalves, 2015), para el logro de las metas que sobre dicho uso se plantea una institución de educación universitaria.
\end{abstract}

Palabras clave: TIC, Uso Didáctico, Educación Universitaria, Afectividad Digital. 


\title{
Uso Didático das Tecnologias da Informação e Comunicação nas Universidades: aspiração ou expectativa?
}

\begin{abstract}
Resumo
O ser humano sempre colocou sua curiosidade e engenho para melhorar sua qualidade de vida, sendo a comunicação uma das áreas mais destacadas devido ao seu notável papel socializador. A convergência dos avanços em eletrônica, informática e comunicações, transmutou-se em telemática e na criação da Internet, que interconectou o mundo e todas as áreas de eventos sociais. A educação, um processo puramente social, tem sido impactada pelo advento da Internet desde os anos noventa, modificando não apenas o tipo de recursos utilizados no processo didático de facilitação e aquisição de aprendizagem, por seus dois principais atores: professores e estudantes, respectivamente; mas também a cultura das instituições de ensino, que, como no caso venezuelano, por imperativo da lei, deve incorporar as Tecnologias da Informação e Comunicação (TIC) em sua missão e planos de desenvolvimento organizacional. Duas décadas após de ter sido establecido como una prioridade o uso da Internet nas instituições de ensino, portanto nas universidades venezuelanas, neste artigo analisamos, baixo a visão dos mundos Popperianos (Popper, 1986), duas instituições universitárias, uma pública e outra privada; para determinar se o uso didático das TIC responde a uma expectativa ou aspiração, e se é pessoal ou institucional. Foi encontrada uma insatisfação com a expectativa legal estabelecida em uma aspiração institucional, dada a existência de uma predisposição negativa generalizada de professores e estudantes para o uso das TIC como recurso para mediar processos didáticos na sociedade atual, destacando o importante papel do componente afetivo pessoal, neste caso da afetividade digital (Goncalves, 2015), para a consecução dos objetivos que sobre esse uso surge duma instituição de ensino universitário.
\end{abstract}

Palavras chave: TIC, Uso Didático, Educação Universitária, Afetividade Digital.

\section{Didactic Usage of Information and Communication Technologies in Universities: ¿aspiration or expectation?}

\begin{abstract}
Human being has always been curious with all its wit about improving its quality of life, being communication one of the most featured areas due to its remarkable socializing role. The convergence of advances in Electronics, Informatic and Communications transmuted into Telematic and the creation of Internet, which has interconnected the world and all social environments. Education, truly a social process, has been impacted by the arrival of the Internet since the 90s, modifying not only the type of resources used in the didactic process of facilitation and acquisition of knowledge involving their two main protagonists: teachers and students, respectively; but also the culture of the educational institutions, which, in the Venezuelan case, by law, must incorporate in their mission and organizational developing plans, the Information and Communication Technologies (ICT). After two decades of establishing the use of Internet as a priority in educational institutions, mostly in Venezuelan universities, in the present article two higher education institutions are analyzed using the Popperian vision (Popper, 1986): one private and one public; to determine if the didactic usage of ICT responds to an expectation or to an aspiration, and as such, if it is personal or institutional. A dissatisfaction of the legal expectation was evidenced, which turned in an institutional aspiration, due to the existence of a negative and general predisposition of teachers and students towards the use of ICT as resources
\end{abstract}


for the mediation of didactic processes in today's society, which highlights the important role of the personal affective component; in this case, digital affectivity (Goncalves, 2015) for the accomplishment of goals that a higher education institution has established.

Keywords: ICT, Didactic Usage, Higher Education, Digital Affectivity.

\section{Esbozo Introductorio}

Desde tiempos inmemoriales, el ser humano, el hombre, en una denominación general, ha sentido interés y curiosidad por entender su entorno. Esa curiosidad ha sido, precisamente, la que le ha permitido acceder a una mejor calidad de vida entre los seres vivos. Ejemplos de ello los encontramos en el descubrimiento de la penicilina, el trasplante exitoso de órganos como el corazón y más recientemente el de pulmones realizado a un reconocido artista venezolano; la producción en serie de bienes materiales como artículos de cuero sin sacrificar animal alguno a partir de la célula de éste, en aplicación de avances en biotecnología (https://www.eleconomista.es/economia/noticias/8136561/02/17/El-cuero-biotecnologico-laalternativa-real-para-quienes-no-quieren-usar-pieles-de-animal.html), o la producción de bienes alimenticios como la carne de pollo sin implicar su sacrificio (https://www.bbc.com/mundo/noticias-45897953, 18/10/2018).

Más, no sólo la curiosidad humana ha permitido alcanzar logros tan importantes en campos como los mencionados previamente, sino también en la invención y actualización permanente de los equipos requeridos para canalizar las comunicaciones entre los miembros de una sociedad, como el telégrafo, la radio, los medios televisivos, el computador, el microcomputador, además de un cúmulo de artefactos que han facilitado sus actividades cotidianas. Ese ingenio humano ha llevado a combinar los avances obtenidos en áreas disciplinares de conocimiento particulares, para generar otros que potencian esos logros individuales, ofreciendo así, mayores beneficios a la sociedad.

Es así como el aprovechamiento de los avances específicos en las áreas de conocimiento referido a las comunicaciones, con la convergencia evolutiva de la electrónica y la informática, devino en la telemática, dando origen a la red de redes, la Internet. Para los televidentes de la década de los años sesenta del siglo XX era cotidiano compartir en familia la tira cómica denominada Los Supersónicos; una familia ubicada en la segunda mitad del siglo XXI (año 2062), en una sociedad que disponía de avances tecnológicos, para la época inimaginables, y que en la actualidad se encuentran en la cotidianidad personal y laboral. Podemos relacionar los mecanismos automatizados para desplazamiento en el hogar y oficina laboral del señor Sónico 
(cintas transportadoras), a Robotina, robot auxiliar en labores del hogar y atención a los miembros de la familia (robots que hoy ayudan a pasajeros a realizar trámites en aeropuertos, procesos industriales y del hogar); la conversación a distancia de Ultra, la señora Sónico (videollamadas, videoconferencias); el hogar "inteligente" de la familia (controles remotos de equipos, teléfonos celulares); incluso el desplazamiento vehicular espacial con autos voladores y estacionamientos informatizados (drones). De este último aspecto, se tiene que, según reseña de prensa del año pasado, la industria automotriz japonesa NEC Corporation, promete sacar al $\begin{array}{llllll}\text { mercado el año } & \text { auto } & 2023\end{array}$ (https://www.japantimes.co.jp/news/2019/08/05/business/car-plane-no-necs-personalquadcopter/\#.XsvM-ERKjIV) .

Con certeza innegable, la creciente curiosidad del hombre produjo a partir de la segunda mitad del recientemente transcurrido Siglo XX, una conjunción de saberes de gran impacto en el quehacer humano en sociedad. Como mencionamos antes, los avances en el campo de las disciplinas informática, telecomunicaciones y electrónica se conjugaron para producir la telemática, y con ello la creación de la red de redes, la red de cobertura mundial: Internet y con ello el surgimiento de un Mundo y Era Digital (Siemens, 2004). Esta red ha propiciado el desarrollo de incontables y diversas aplicaciones para ser utilizadas en prácticamente todos los ámbitos de desarrollo social, y por ende en la educación (Aula virtual, Blog, WhatsApp, Google, Hangouts, Correo Electrónico, Redes Sociales).

Es evidente como, desde la aparición de la Internet en la década de los noventa, la convivencia en sociedad ha experimentado grandes y constantes cambios en los distintos órdenes. Actualmente, es difícil concebir las interrelaciones personales y sociales sin la intermediación de la red de redes; escuchar diálogos en los que se mencionan las redes sociales, la nube, y la realización de trámites en el gobierno y comercio electrónico; es ahora habitual y cotidiano. Muchos habitantes son miembros de la cibersociedad (Joyanes, 1997), son eciudadanos.

Siendo así, es natural que las diversas herramientas de la web hayan impactado la educación como complejo proceso social y, en consecuencia, el quehacer de sus actores protagónicos: educadores y educandos, no importando si son nativos o inmigrantes digitales (Prensky, 2001). De modo que a estos actores se les exige desarrollar competencias, habilidades, destrezas para utilizar las herramientas digitales en sus procesos de facilitación, 
gestión y autogestión de aprendizajes, respectivamente. El docente se ve requerido a administrar el diseño curricular de su área de conocimiento utilizando dichos recursos para mediar su proceso de facilitación de aprendizajes, y el estudiante a desarrollar competencias para auto y cogestionar conocimientos en forma colaborativa y cooperativa. Esta realidad se encuentra presente en todos los niveles del sistema educativo formal, siendo el contexto universitario el que denota una característica especial, por ser la encargada de formar los profesionales requeridos por la Nación y la sociedad para su desarrollo y progreso.

\section{Comprensión Filológica de la Expectativa y la Aspiración}

En el contexto universitario, Subsistema de Educación Universitaria en el caso venezolano, la inserción de las TIC en sus diversas actividades y con especial énfasis en la mediación de sus procesos didácticos, ha generado un cúmulo de aspectos que giran en torno a la posibilidad de lograr, en una misma institución, la coexistencia de diversas modalidades educativas, con diversos niveles de virtualización cónsonos con la naturaleza de las carreras y unidades curriculares. Lo anterior puede denotar un cúmulo de expectativas o aspiraciones por parte de sus actores sociales, los cuales no se enmarcan exclusivamente en docentes y estudiantes, involucra, además, a sus gerentes (coordinadores, directores, decanos, vicerrectores y rectores, entre otros) los cuales constituyen el eje motor para la formulación y ejecución de políticas internas.

Indagando el significado de la palabra expectativa, encontramos que la misma proviene del latín exspectātum 'mirado, visto', y su significado es, según el Diccionario de la Real Academia Española, esperanza de realizar o conseguir algo, posibilidad razonable de que algo suceda (https://www.rae.es/dpd/expectativa). Esa esperanza o posibilidad puede ser de orden individual, personal, tal y como es alcanzar un título profesional, un grado académico; o de orden colectivo, social, si se refiere a una realización que trasciende lo personal porque es deseado o esperado por un grupo social de rango local, regional, nacional o tal vez internacional. Entonces, ¿qué es una expectativa social? Para los autores, una expectativa es un DEBER SER. El precitado Diccionario de la Real Academia define la palabra deber como un verbo, es decir, como una acción que connota "Aquello que se tiene la obligación de hacer" (https://dle.rae.es/deber?m=form); en sentido general, aquellas acciones que la sociedad, como entidad aglutinadora de ciudadanos, espera que se cumpla, que se realice para obtener los 
beneficios individuales y colectivos esperados de la puesta en práctica de los servicios, normas, reglamentos, leyes que rigen la convivencia armónica entre sus miembros.

En consecuencia, tenemos que la distinción entre una expectativa social o una aspiración institucional decanta en la trascendencia de aquello que se va a realizar, no es lo mismo "querer" hacer, que "deber" hacer; de modo que se pudiera pensar que el uso de las TIC en todos los órdenes de la sociedad es una expectativa. Además, la mencionada obligación del deber ser que reflejamos en el párrafo anterior está contenida en las leyes, normas, estatutos, decretos, en los corpus teóricos derivados de procesos de investigaciones, en diseños curriculares; en suma, en lo que Popper (1986) denomina el mundo de los productos mentales.

\section{Ámbito del Mundo del Deber Ser}

En el contexto venezolano, el Estado ha promulgado e implementado una serie de políticas públicas que persiguen utilizar todos los componentes esenciales de la plataforma tecnológica para el desarrollo de las TIC en todos los ámbitos de la sociedad, incluyendo el educativo. Políticas que tienen su sustento normativo en los artículos 102, y 103 de la Constitución de la República Bolivariana de Venezuela (1999), los cuales son claros y taxativos al establecer la educación como un derecho humano y bien social, así como el rol docente del Estado Venezolano. Además, el artículo 108 de la precitada Carta Magna establece la inserción de las tecnologías y de los medios de comunicación en esa relación Educación-Estado Venezolano.

Dentro del conjunto de políticas promulgadas, el 10 de mayo del año 2000, como parte del Plan Nacional de Telecomunicaciones 2000-2011, fue promulgado por el Estado Venezolano el Decreto 825, con el cual se declara el acceso y el uso de Internet como política prioritaria para el desarrollo cultural, económico, social y político de la República Bolivariana de Venezuela, según publicación en la Gaceta Oficial No. 36.955 de la Presidencia de la República (http://www.conatel.gob.ve/wp-content/uploads/2017/01/sobre-internet.pdf). En este Decreto se incluye como objetivo de la Cultura Digital en el país, fomentar la investigación científica y tecnológica en los diferentes niveles educativos (escuelas, colegios, universidades e institutos de ciencia) y en sectores privados, es decir, se decreta el uso educativo de la Internet en las instituciones escolares. En sus Artículos 5, 7 y 8 se destaca lo siguiente:

Artículo $5^{\circ}$ : El Ministerio de Educación, Cultura y Deportes dictará las directrices tendentes a instruir sobre el uso de Internet, el 
comercio electrónico, la interrelación y la sociedad del conocimiento. Para la correcta implementación de lo indicado, deberán incluirse estos temas en los planes de mejoramiento profesional del magisterio. (Resaltado nuestro)

Artículo $7^{\circ}$ : El Ministerio de Educación, Cultura y Deportes, en coordinación con los Ministerios de Infraestructura, de Planificación y Desarrollo y, de Ciencia y Tecnología, presentará anualmente el plan para la dotación de acceso a Internet en los planteles educativos y bibliotecas públicas, estableciendo una meta al efecto.

Artículo $8^{\circ}$ : En un plazo no mayor de tres (3) años, el cincuenta por ciento $(50 \%)$ de los programas educativos de educación básica y diversificada deberán estar disponibles en formatos de Internet, de manera tal que permitan el aprovechamiento de las facilidades interactivas, todo ello previa coordinación del Ministerio de Educación, Cultura y Deportes.

Como se observa en el Artículo 8, en dicho Decreto se establecía una temporalidad de 3 años, es decir, para el año 2003, el cincuenta por ciento (50\%) de los programas educativos de los niveles precedentes al universitario deberían haber estado disponibles en formatos de internet. Lo anterior representa una clara evidencia de la importancia que para el Estado Venezolano ha tenido la virtualización de la educación en el país.

Cuatro años después se promulga el Decreto 3390 (2004), sobre el uso de software con estándares abiertos (también conocido como software libre) en la Administración Pública Nacional, del cual desprendemos dos acciones a destacar. La primera tiene que ver con el Proyecto Canaima Educativo, que inició en el año 2009 y constituye una respuesta al mencionado Decreto 3390 (2004), el cual se inició en el primer grado de educación primaria y progresivamente en los grados siguientes y precedentes al subsistema universitario. La segunda consideración se ubica en el contexto universitario y el empleo de las Plataformas Virtuales de Aprendizaje (PVA) como herramientas de gestión de los procesos didácticos, las cuales permiten alojar, diseñar y gestionar aulas virtuales como apoyo a la presencialidad, en modalidad mixta o virtual; las Instituciones de Educación Universitarias (IEU) fueron incorporando Moodle para tal propósito y en correspondencia con el Decreto sobre el uso del software libre.

También, en el año 2009, se promulga la Ley Orgánica de Educación, la cual establece en su artículo 15 los fines de la educación, contemplando la formación dentro de una perspectiva 
integral, mediante políticas de desarrollo humanístico, científico y tecnológico, vinculadas al desarrollo endógeno productivo y sustentable. En el contexto universitario, es necesario mencionar, una propuesta de especial relevancia que, hasta la fecha de redacción del presente artículo, no ha sido promulgada por las máximas autoridades del Ministerio del Poder Popular para la Educación Universitaria (MPPEU). Nos referimos a la Propuesta de Normativa Nacional para la Educación Superior a Distancia (2009), siendo la última versión la presentada a finales del año 2012, la cual recibe el nombre de Propuesta de Normativa para la Educación Universitaria a Distancia (2012).

Como es natural, al estar previsto en la Carta Magna (1999) y en la Ley Orgánica de Educación (2009), las IEU venezolanas están obligadas a utilizar las herramientas tecnológicas digitales en las actividades que le son inherentes como son la docencia, la investigación y la extensión, además de la gestión administrativa. Es por ello que los autores, encontrándonos a finales de la segunda o a principios de la tercera década del siglo XXI, año 2020, inmersos en un contexto socioeducativo diferente al de los años de promulgación del mundo normativo (Popper, 1986) mencionado en los párrafos anteriores, consideramos relevante e importante reflexionar sobre la praxis institucional en este uso didáctico de las TIC en el contexto universitario, tomando como ámbito de análisis (estudio) la función docencia en dos instituciones universitarias venezolanas de distinta naturaleza: una autónoma y otra de gestión privada, ambas ubicadas en la región central del país. Para ello, tomamos como generadoras de dicho análisis las interrogantes: el uso didáctico de las TIC, ¿es realmente una expectativa legal? Si lo fuese, ¿quién es o quiénes son los expectantes?, ¿Está satisfecha esa expectativa? De no ser una expectativa, ¿cómo puede calificarse? En las próximas líneas trataremos de dar respuesta a estas interrogantes.

\section{Ámbito del Mundo Físico}

Dentro de su marco de perenne transformación, las TIC han permitido superar condiciones de espacio y tiempo, ofreciendo una amplia gama de recursos interactivos, favoreciendo la flexibilidad que algunos sistemas educativos formales necesitan, convirtiéndose así en propuestas importantes de innovación e inclusión. La conjunción de la informática, la telemática y las comunicaciones obliga a las IEU a promover su uso en todas las actividades vinculadas a la docencia. Obligación que debe responder de manera taxativa, al marco jurídico 
promovido por el Estado Venezolano para tal fin y del cual ya hemos disertado de manera sintetizada.

En correspondencia con lo descrito en el apartado anterior, cada IEU del país ha engranado esfuerzos en aras de dar respuesta al ámbito normativo de la nación, presentando así un contexto físico donde cada universidad pretende ubicarse y adaptarse. Esfuerzos que se ubican no sólo a nivel nacional, sino también latinoamericano; es así como un estudio efectuado por la UNESCO (2006), se basó en un informe sobre la Educación Superior en América Latina y el Caribe, que de manera conjunta con el Instituto Internacional para la Educación Superior en América Latina y el Caribe (IESALC), recopilaron datos de cada uno de los países que integran esa región, basándose en estudios propios realizados por investigadores de diversas universidades. Entre ese grupo de investigadores se encontraron Cursi (2005) y Facundo (2005), quienes en conjunto, aportaron características actuales de la educación, problemática vivida en cada país, y a partir de esa información, elaboraron el informe que muestra la realidad de Latinoamérica a fin de desarrollar modelos que permitan el mejoramiento continuo y la elevación del bajo nivel relativo al alistamiento digital; buscando así la inserción de los recursos tecnológicos disponibles como un medio para alcanzar la calidad educativa.

En dicho informe se parte de los roles que debe desarrollar el docente universitario, como una de las piezas fundamentales de la educación. Roles que tal y como lo menciona Facundo (2005), además de las actividades de docencia, investigación, extensión y administración; su tendencia actual es al empleo de tecnologías digitales de información y comunicación en los diferentes procesos y funciones. Además, en dicho informe se establecieron cuatro niveles en función de la naturaleza técnico-pedagógica, aplicables tanto a la digitalización de la educación a distancia como a la presencial, para que de una manera sencilla y rápida se pudiera conocer la realidad del país a estudiar, y saber cuáles ámbitos se deben mejorar para alcanzar la educación virtual ideal.

Considerando a Facundo, se pueden resumir los cuatro niveles de la siguiente manera: Nivel I: emplean el computador y otros medios digitales principalmente como vehículo de presentación y entrega de contenidos. Nivel II: destaca el uso de herramientas digitales en docencia y se evidencia la virtualización de otras funciones, generalmente en tareas administrativas: inscripción de estudiantes, reportes de notas y certificaciones en línea. Esta etapa es la virtualización de la educación, ya que no se aprecian cambios estructurales en el 
currículo o en las pedagogías. Nivel III: incorporan el uso de plataformas virtuales para digitalizar e integrar las diversas funciones de la educación universitaria. En docencia hay mayor uso de herramientas digitales, particularmente audiovisuales, pero todavía dentro de modelos pedagógicos e institucionales, el currículo permanece lineal y sin flexibilidad. Nivel IV: además del uso de plataformas virtuales que integran las diversas funciones universitarias, se aprecian estructuras curriculares multi-ruta y modelos pedagógicos auto-dirigidos, que es lo que diferencia este nivel del anterior.

Un aspecto a destacar en estos niveles, es que los cambios realmente significativos desde el ámbito curricular sólo están presentes en el nivel IV. Tanto Cursi (2005), como Facundo (2005), coincidían al momento de efectuar sus estudios, que todavía no se disponía en Latinoamérica de auténticas instituciones y programas de educación superior virtual que alcanzaren el nivel IV. Al respecto, señalaron que el mayor porcentaje de instituciones se encontraban apenas en lo que se ha denominado como proceso de virtualización de la educación, es decir, en los niveles I y II.

A pesar de que estos estudios datan de más de una década, es acertado señalar en el caso venezolano, que no todas las IEU están en el mismo nivel de integración digital y para llegar al último nivel, se requiere de emplear las TIC de manera sistemática, organizada y racional en toda la estructura organizacional de las referidas instituciones. Estos niveles taxonómicos permiten analizar la realidad de cada institución universitaria y clasificarla de manera sencilla; revisando la evolución y perspectiva de la educación virtual. Un breve diagnóstico de cada IEU venezolana puede basarse muy bien, en establecer indicadores para cada nivel y en función de ello, determinar en qué posición de "integración digital" se encuentran, pues en correspondencia con el ámbito del deber ser, todos los esfuerzos de las IEU deberían estar enfocados en subir, de manera progresiva, en cada uno de estos niveles de virtualización de la educación universitaria.

De modo general, para ilustrar el uso educativo de las TIC, se describe el contexto específico de una Institución de Educación Universitaria de Gestión Privada (en adelante IEUGP), ubicada en la región central del país. En el año 2004, seis años después de ser autorizada por el Estado Venezolano para compartir su responsabilidad en cuanto a la provisión del derecho humano y bien social que constituye la educación y cuatro años después del Decreto 825 (2000), esta institución de gestión privada creó dentro de su estructura organizacional la Dirección General de Nuevas Tecnologías (en adelante DGNT), para administrar los esfuerzos 
en el desarrollo de la infraestructura informática necesaria para impartir educación virtual, mediante la puesta en práctica de un sistema de gestión de aprendizajes, con base en el software libre Moodle, al cual se denominó Acrópolis. Esta fue una decisión institucional de avanzada y de decidida intención de cumplir la corresponsabilidad asignada por el Estado Venezolano en cuanto al uso educativo de las TIC, para tener disponible el servicio educativo provisto en formato de internet.

Desde entonces, esa Dirección se ha responsabilizado por el entrenamiento a los docentes de pregrado y postgrado, en el uso de la mencionada plataforma de aprendizaje. Se dictan talleres, cursos que inicialmente fueron concebidos bajo la modalidad presencial, con sesenta (60) horas de duración, pero debido a la poca receptividad del profesorado hacia este entrenamiento con argumentos de la poca disponibilidad de tiempo para asistir a las sesiones planificadas, se cambió a la modalidad semipresencial o combinada, además de reducir la duración a diez y seis (16) horas, ocho presenciales y ocho virtuales, con la aplicación de la estrategia didáctica aprender haciendo. El criterio de aprobación de dicho curso es el diseño del aula virtual de la unidad curricular bajo responsabilidad del docente o grupo de docentes que comparten esa facilitación de aprendizajes.

Otra decisión gerencial para propiciar la socialización del uso educativo de las TIC en la referida institución, fue la de acreditar como Trabajo de Ascenso en el escalafón universitario el diseño del aula virtual de la unidad curricular facilitada por el o los docentes (hasta máximo dos docentes por trabajo de ascenso), previo cumplimiento de todos los requisitos exigidos, entre ellos la presentación del certificado del Curso de Formación Docente a los docentes que aspiren ascender a partir de la categoría de Profesor Agregado, la presentación del plan de trabajo ante el Consejo Universitario para su aprobación y consecuente entrega del informe correspondiente en el que se plasme el seguimiento de todos los criterios metodológicos y de diseño que conlleva un aula virtual, así como de los criterios científico-técnicos de una investigación aplicada.

Así mismo, se han ejecutado decisiones de orden financiero al pagar honorarios profesionales equivalentes a treinta y dos (32) horas, en el escalafón que ostente el docente, por el diseño de cada aula virtual. Además, en el plan de estudios del Curso de Formación Docente (requisito indispensable para ascender en el escalafón universitario) se incluye la unidad curricular Diseño Instruccional Computarizado, cuyo requisito de aprobación es el diseño de las 
dos primeras unidades temáticas del programa analítico bajo responsabilidad del docenteparticipante. También, se asignó un grupo de cinco docentes con experiencia en el diseño e implantación de aulas virtuales que, voluntariamente, manifestaron su disposición para contribuir en la formación de sus pares académicos en el uso de estrategias virtuales y acompañamiento docente en cada facultad y dirección general, mediante la facilitación de horas semanales, fuera de su carga docente institucional. Todas estas decisiones se tomaron bajo la premisa de ser favorecedoras de la incorporación de las TIC como apoyo a la presencialidad.

Más, en referencia a las IEUGP, se tiene que, en las conclusiones del Simposio titulado: “Uso de las TIC en las Instituciones de Educación Universitaria de Gestión Privada", realizado en la Facultad de Ciencias de la Educación de la Universidad de Carabobo en el marco de la Undécima Expedición EDUWEB 2017, conocida la experiencia de cuatro de las cinco instituciones que operan en la región carabobeña, se destacaron varios aspectos, los cuales se resumen a continuación. Estas instituciones hacen esfuerzos institucionales para acatar la sugerencia de la UNESCO de utilizar las TIC; aplican una concepción integral del uso de la tecnología para apoyar el servicio educativo que ofrecen, es decir, en las actividades de docencia, investigación, extensión y gestión administrativa. El uso de las TIC es fundamentalmente de apoyo a la modalidad presencial, dado que, para el mes de octubre 2017, fecha de realización del Simposio, no estaba todavía aprobada la autorización del Estado, mediante la normativa que regule dicho uso.

Las aulas virtuales, correo electrónico y redes sociales son herramientas tecnológicas de uso común en estas instituciones. La experiencia en cada una de las cuatro instituciones participantes, ha permitido verificar la idoneidad del uso de las TIC para superar las dificultades derivadas del contexto país en el pasado reciente. Además, destacaron que las deficiencias presupuestarias de las IEUGP en un contexto país de economía inflacionaria, limitan la inversión en el desarrollo de aplicaciones educativas de las TIC.

Otras conclusiones del mencionado Simposio están referidas al desconocimiento existente en facilitadores y estudiantes sobre las bondades de las TIC para aprender y mediar en la Era de la Información. Los patrones mentales, resistencia al cambio de facilitadores y estudiantes son obstáculos para aplicar estrategias virtuales de aprendizaje; y como fortaleza se tiene las habilidades y destrezas desarrolladas por el estudiante debido al uso lúdico de la tecnología, lo cual propicia el uso educativo de las TIC. 
Por último y no menos importante, destacaron que se hace necesario el apoyo del Estado Venezolano en sus Políticas Públicas para garantizar una buena conectividad y con ello el uso educativo de las TIC. Se consideró igualmente necesaria la aprobación del Reglamento de Educación a Distancia, o la autorización el entonces Ministerio del Poder Popular para la Educación Universitaria, Ciencia y Tecnología, para aplicar la modalidad virtual. Por cuanto el uso de las TIC en las IEUGP es una herramienta organizacional para contribuir con la Nación a lograr objetivos del desarrollo sostenible para 2030 (2015), relacionados con la inclusión, la equidad y el aprendizaje permanente para todos.

Se denota así cómo en el ámbito físico de estas instituciones universitarias, se han desarrollado importantes y notables avances con el fin de tratar de cumplir con el mundo normativo establecido en el ámbito del DEBER SER. No obstante, la diversidad de situaciones que se suscitan a nivel del micro currículo en cada subsistema educativo y, específicamente en cada institución, complejizan aún más la inserción de recursos tecnológicos, derivado de la variedad de factores intervinientes y la diversidad de aristas que se desprenden como consecuencia de pretender su inserción organizacional.

\section{Ámbito del Mundo del Ser}

En la sección anterior se mencionó el caso específico de una IEUGP de la región central del país y cómo parte de sus políticas internas, pretenden responder efectivamente al mundo normativo que el Estado Venezolano ha promulgado en las últimas décadas. Es importante mencionar que dicha institución no está autorizada para impartir educación a distancia virtual, por ello ha promovido el uso de estrategias virtuales como apoyo a las actividades presenciales, para superar las dificultades cotidianas de docentes y estudiantes derivadas de las debilidades societales en materia de seguridad y transporte (contexto inseguro y escaso transporte, respectivamente). Así, se redujo, temporalmente, el horario laboral administrativo y se programó el último bloque horario nocturno (8:55 a 10:25 p.m.) para ser cumplido con apoyo de estrategias virtuales. Para lograr esa meta fueron aprobadas en la sesión ordinaria del Consejo Universitario No. 535 de fecha 8 de agosto de 2018, las Normas que Regulan las Actividades Académicas de Apoyo a la Presencialidad, en las cuales se estableció una carga horaria virtual para las unidades curriculares teóricas o teórico-prácticas equivalente hasta un cincuenta por ciento $(50 \%)$ de la carga crediticia de dicha unidad curricular. 
Además, en estricta aplicación de los criterios tecnocurriculares que regulan el diseño, cambio e innovación educativos, la IEUGP tomó, como primera decisión para propiciar el éxito en la aplicación de estas estrategias, entrenar al personal docente, tanto de pregrado como de postgrado, con un Curso Taller denominado Formación de Tutores Virtuales; responsabilidad que fue asignada a la antes mencionada DGNT. Los docentes que asisten al curso Formación de Tutores Virtuales, diseñan las dos primeras unidades programáticas del aula virtual de la unidad curricular bajo su facilitación, requisito de aprobación del mencionado Curso, pero no completan su diseño; por ende, generalmente no emplean el Aula Virtual para apoyar sus actividades de docencia presencial. Si una de las fortalezas del uso de las TIC en el contexto socioeducativo actual es precisamente de orden económico para el docente y el estudiante al evitarse costos de transporte, además de la seguridad en el traslado, surge en los autores la inquietud ¿por qué no se motivan a diseñar completamente su aula virtual?

Una rápida mirada a las estadísticas que posee la DGNT, suministradas por su autoridad directiva, nos evidencia que para el período lectivo 2019-3CR, lapso 4-11-2019 a 19-03-2020, se encuentran parcialmente diseñadas (como se indicó, sólo tienen al menos dos unidades temáticas con material didáctico y actividades instruccionales cargadas) y no aprobadas por el Consejo Universitario, dieciocho (18) aulas en la Facultad de Ciencias Sociales integrada por cuatro escuelas, 8 en la Facultad de Ciencias de la Educación, 7 en la Facultad de Ciencias de la Salud, en la Facultad de Ciencias Jurídicas y Políticas también 7, veintiuno (21) en la Dirección General de Estudios Básicos y Generales, y en la Facultad de Ingeniería, integrada por 7 escuelas un total de sesenta y siete (77).

Ahora bien, en lo que se refiere a las aulas diseñadas, con el guion instruccional aprobado por el Consejo Universitario, y por tanto completamente planificadas con estrategias virtuales, las cifras se reducen y se tienen: 4 de la Dirección General de Estudios Básicos y Generales, 6 en la Facultad de Ciencias Sociales, diez (10) en la Facultad de Ciencias de la Educación y 6 en la Facultad de Ingeniería. Aunado a lo anterior, se constató que algunas de esas aulas tienen una data de creación del año 2005 y 2008, siendo las más recientes del año 2017. Es importante, además, contrastar ese número con el total de unidades curriculares o asignaturas que integran cada carrera, según disposiciones curriculares en materia de Educación Universitaria, el cual asciende a 50 en promedio. 
Razones de esta realidad la encontramos en la respuesta de la docente responsable por la planificación y ejecución del curso Formación de Tutores Virtuales, quien a la pregunta ¿A qué factores atribuye que la institución no ha podido lograr el objetivo de incorporar activamente a los profesores a las aulas virtuales como apoyo a la actividad presencial? respondió:

El personal docente puede emplear diversas herramientas virtuales (Aulas Virtuales, Blogs, Google Site, Google Classroom, Hangouts, Skype, Correo Electrónico, entre otros) es decir, no hay una condición obligatoria para usar el aula virtual como estrategia didáctica.

Los profesores que se postulan en los talleres semipresenciales o en línea al aprobar el curso egresan con un $40 \%$ del aula virtual diseñada, esto significa que requiere un tiempo adicional a la labor académica para consolidar el producto final, tiempo que no están dispuestos a dar a la institución, señalando además con actitud mezquina que las aulas les pertenecen a la institución y no al que las diseñó.

Los profesores manifiestan en los talleres la necesidad de disponer de un espacio físico para facilitar el acceso a la labor académica de apoyo a la actividad presencial, porque no poseen un computador con acceso a internet.

En algunas ocasiones los profesores presentan debilidades en el manejo de las estrategias de aprendizaje distintas al programa de estudio que imparten; esta situación interfiere al asignar actividades didácticas en el aula virtual.

En ocasiones los profesores manifiestan que realizar la labor académica en las aulas virtuales requiere mayor inversión de tiempo, además de las fallas del servicio eléctrico y la conectividad.

Las facultades a través de los coordinadores curriculares deben solicitar al personal docente un informe de las actividades de apoyo a la presencialidad, pero para lograrlo se debe implementar una normativa que regule, supervise, el control y seguimiento de las actividades académico-administrativas planificadas y cumplidas.

La institución debe disponer de un equipo multidisciplinario para fortalecer la gestión de aulas virtuales para que los expertos en contenido puedan apoyar la labor académica del personal docente.

(Correo electrónico enviado por docente formadora a autor, 04/02/2020, $14: 35)$

Al analizar estas respuestas observamos factores, en su mayoría, de índole individual del docente aun cuando, también, hay implicaciones institucionales, como son la falta de monitoreo, de acompañamiento virtual al docente, la conformación de un equipo de expertos en contenido y en diseño instruccional, entre otros. No obstante, debe señalarse que en la referida IEUGP, en 
trabajo mancomunado con su Coordinación de Redes y Telecomunicaciones, se han destinado dos laboratorios de informática a disposición del personal docente para realizar las acciones administrativas y académicas que requieran el apoyo logístico computacional, pero los mismos son empleados por los docentes, fundamentalmente, para la transcripción de calificaciones y cierre de actas, no como apoyo virtual a las actividades didácticas presenciales.

Cabe destacar que, uno de los autores de este artículo se desempeñó como facilitador en el entrenamiento al personal docente. Desde el Rectorado de la institución se le hizo entrega del horario correspondiente con la lista de profesores participantes en el taller. Se le informó que el Decano de la facultad había notificado a sus docentes la importancia de la actividad y que éstos habían manifestado su disposición a participar. Más la realidad fue:

\section{[...] al llegar a los laboratorios asignados NINGÚN} PROFESOR LLEGÓ. Le manifesté mi obvio descontento al decano pues no era la primera vez que sucedía. Lo que logró decirme (tal vez como buscando excusar a los docentes) fue que los horarios de clase se lo impedian, que siempre andaban muy ocupados con otras labores en la universidad. (Conversación autor-docente formadora, 2020)

Lo descrito en los párrafos anteriores, aunado a la inasistencia de los docentes a las horas de formación pautadas por la DGNT o con sus pares académicos voluntarios para cooperar en su entrenamiento como tutores virtuales, puede ayudar a comprender, por ejemplo, la solicitud elevada al Consejo Universitario de esta institución, de eliminar la carga virtual en una unidad curricular de carácter teórico, por parte de docentes de dilatada trayectoria y de la tercera edad no familiarizados con las TIC, bajo el argumento siguiente:

[...] los contenidos programáticos de las mencionadas asignaturas fueron diseñados para el modo presencial, es evidente que su presentación y discusión en persona, alumno-docente, resulta en mejor comprensión y manejo de los conceptos e interrelaciones de información de elevada importancia e interés. (Correspondencia de fecha 18/11/2019)

Es necesario informar que este docente, a pesar de su trayectoria laboral de años en la Institución, no ha realizado alguna de las actividades planificadas para entrenar a los profesores, tal vez porque como lo indicó una docente formadora en el diseño de aulas virtuales: "muchos profesores no veían cómo usar aulas virtuales los ayudaría en sus profesiones" (Conversación 
docente-autor), o porque "muchos de esos docentes no se tomaban esta formación en aulas virtuales en serio" (Conversación docente-autor).

Más, la educación virtual no consiste en trasladar la planificación presencial a un formato digital. Por el contrario, se trata de aprovechar las fortalezas de la comunicación asíncrona para lograr aprendizajes y la flexibilidad espacio-tiempo que estos entornos promueven. Ello requiere el dominio del docente no sólo del área de conocimiento bajo su responsabilidad sino de teorías sobre el aprendizaje, diseño de la instrucción, manejo de la herramienta a utilizar, así como la disposición del estudiante para aprender de manera autónoma, entre otros aspectos.

La misiva referida en los párrafos precedentes cierra con la premisa de que con la educación presencial se ofrece a los estudiantes una mejor y más completa docencia. El argumento esgrimido nos indica el desconocimiento en materia de educación asistida por recursos telemáticos, así como de la gama de medios digitales para enriquecer la docencia universitaria, disponibles a finales de la segunda década del siglo XXI. Por otra parte, nos preguntamos, ¿acaso la educación con apoyo virtual es peor e incompleta? ¿Es, entonces, acertada la expresión de Marín y Cabero (2010) al afirmar que las instituciones universitarias son del siglo XIX, los docentes del siglo XX y los estudiantes del siglo XXI? En este orden de ideas, debemos informar que al preguntar a un estudiante de la IEUGP si consideraba que los estudiantes eran capaces de aprender por sí mismos con la orientación recibida en un aula virtual respondió negativamente, debido a la necesidad que se tenía de interactuar con el docente a los fines de comprender los procesos inherentes a la unidad curricular de su área de formación, jurídica en este caso específico.

En consecuencia, predominan de manera notable y destacada, los esquemas presenciales tradicionales y magistrales de la docencia universitaria, en la cual el docente imparte el conocimiento y el estudiante, cotidianamente llamado alumno, lo recibe, generando un proceso comunicativo unidireccional, impropio de la Sociedad de la Información y del Conocimiento, lo cual desdice mucho de la realidad social actual, caracterizada por la hiperconexión y la diversidad de herramientas tecnológicas disponibles para mediar procesos didácticos.

Pudiera aducirse que la disyuntiva presentada en el ámbito de los esquemas mentales de docentes y estudiantes reside, en el caso del presente documento, por el hecho de hacer referencia, exclusivamente, a una IEUGP. No obstante, a continuación describiremos datos y 
estudios efectuados en una Universidad Nacional, ubicada también en la región central del país, la cual formó parte de las mesas de trabajo organizadas por la Oficina de Planificación del Sector Universitario (OPSU) para el diseño del Proyecto Nacional de Educación a Distancia, iniciado a mediados del año 2007 y llevado a cabo por el Ministerio del Poder Popular para la Educación Superior (actualmente Educación Universitaria).

En las referidas mesas de trabajo se contemplaron aspectos vinculados a la formación de docentes y estudiantes, diseño de cursos, tecnología y normativa; siendo uno de sus principales resultados la Propuesta de Normativa para la Educación Universitaria a Distancia, de la cual ya hicimos alusión en la sesión correspondiente al mundo normativo. En los años siguientes, la referida Universidad Nacional realizó proyectos pilotos para el uso adecuado de las tecnologías en ambientes educativos reales, con la finalidad de medir el beneficio y el impacto de esta modalidad en su realidad universitaria; para lo cual dispone de una Dirección de Tecnología Avanzada (DTA) que realiza la coordinación de cada proyecto conjuntamente con la Dirección TIC de cada Facultad.

Es así como, a modo ilustrativo, tenemos el caso de una experiencia ejecutada en una de sus Facultades, basada en un Curso Introductorio cuyo objetivo fue promover el acceso a un grupo de bachilleres que habían sido excluidos de la Educación Superior, proponiendo mejorar los procesos de inclusión con aspectos de calidad y cambios pedagógicos. Todo ello, a través de la realización de diferentes actividades programadas bajo la modalidad de Educación a Distancia y con apoyo de las TIC, a fin de concienciar a todos los involucrados en la filosofía educativa que se imparte en esta institución, procurando cumplir a cabalidad los lineamientos de la educación universitaria venezolana. (Proyecto Génesis, 2009). A pesar de que este Proyecto no presentó continuidad, motivado a diversos factores, el mismo representó un cambio innovador en la institución, pues incrementó en la comunidad estudiantil y profesoral la necesaria formación y preparación en el uso didáctico de los recursos tecnológicos de avanzada (Goncalves, 2009). Estas aseveraciones se realizaron ya que uno de los autores del presente artículo se desempeñó como miembro de la mesa técnica del precitado proyecto y, además, coordinador de una asignatura de su Curso Introductorio.

Si bien, el proyecto precitado fue a nivel de pregrado, en el ámbito de postgrado de la precitada institución (Goncalves, 2011) también emergen evidencias de que la integración curricular de las TIC en la educación universitaria es un proceso complejo, por la diversidad de 
factores involucrados. Es así como, una experiencia previa centrada en la alfabetización de los profesores del postgrado de la facultad referida (Páez, 2008), dejó planteada la necesidad de estudiar el papel de factores intervinientes como la motivación, entusiasmo, actitud hacia la educación asistida por Internet y la presencia de patrones mentales tradicionales, lo cual pudo haber influido en la deserción que se presentó en esta experiencia. De manera incuestionable, un elemento que cobra rol protagónico en estas experiencias sistematizadas (Páez y Arreaza, 2013; Arreaza, Páez y De Sousa, 2013), se vincula directamente a la afectividad de los corresponsables del acto didáctico.

Se percibe, entonces, como cada institución universitaria, ya sea pública o privada, en atención al mundo normativo venezolano en el cual se insertan y, atendiendo además a sus propias particularidades funcionales y del contexto inmediato; han desarrollado una serie de acciones y políticas internas; que en términos generales tienden a ser análogas en cuanto a los procedimientos y resultados obtenidos. Resultados que generalmente evidencian la resistencia al cambio de algunos actores clave en estos procesos de innovación universitaria, es así como Goncalves (2018) indica que dicha resistencia es común en este tipo de instituciones, por cuanto la cultura tecnológica de la educación universitaria tradicional puede obstaculizar el desarrollo de experiencias educativas innovadoras, ya que prevalece una predisposición no favorable al uso de algunos recursos digitales.

\section{¿Expectativa o Aspiración Institucional?}

En las secciones anteriores hemos presentado los tres ámbitos que explanan los mundos Popperianos (Popper, 1986), y las expectativas o aspiraciones de logro al incorporar las TIC en las IEU, para garantizar su uso didáctico de manera efectiva y pertinente. Se percibe de manera diáfana, como en los casos de las instituciones universitarias a las cuales se hizo referencia en el presente artículo y, considerando los niveles taxonómicos de la educación virtual universitaria presentados por Facundo (2005); que dichas instituciones cumplen con los niveles I y II, escasamente presentan algunos rasgos del nivel III.

Si bien la normativa nacional que rija los estudios universitarios a distancia no está promulgada por las autoridades competentes, son evidentes las notables acciones que las instituciones universitarias realizan en adecuar sus estructuras y funcionamientos, para insertar el uso didáctico de las TIC, ya sea como apoyo a la presencialidad o bajo modalidades mixtas. Entonces, la aspiración institucional es más que evidente, tanto el Estado Venezolano como las 
IEU han realizado esfuerzos para este fin; sin embargo, la disyuntiva está presente en la expectativa social, específicamente en los docentes y estudiantes que hacen vida en las universidades. La aspiración institucional permite percibir que las universidades presentan algunos rasgos de una organización digitalmente inteligente, para lo cual Goncalves (2015) indica que una organización digitalmente inteligente se caracteriza por el uso inteligente de la tecnología digital, funcionando como una estructura integrada que trabaja de manera sinérgica en un todo, en un proceso que no implica modificar todas sus estructuras, sino aprender a gestionarlas bajo parámetros diferentes.

Sin embargo, el análisis no puede limitarse al ámbito organizacional, es necesario dirigir la mirada reflexiva a los responsables del acto didáctico: docentes y estudiantes; ya que constituyen el marco de acción real y puesta en práctica de los mundos físico y normativo, aludiendo a la concepción Popperiana (Popper, 1986). Esto nos permite comprender si realmente existen expectantes y quiénes son; ya que además de los aspectos aptitudinales referidos a la formación y preparación, tenemos los aspectos actitudinales que desencadenan en estos actores, un conjunto de acciones y hechos que de manera indefectible pueden favorecer o inhibir la inserción efectiva de las TIC en la didáctica universitaria. Estamos en presencia de lo que Goncalves (2015) intitula como Afectividad Digital, para lo cual menciona que se trata del conjunto de sentimientos, emociones y pasiones de una persona, vinculados con los aspectos actitudinales y volitivos que en el contexto educativo se estudian bajo el contexto de la motivación. Además, Goncalves señala que el componente afectivo del docente y del estudiante se interrelacionan, en un proceso de realimentación constante, en el que la motivación y la corresponsabilidad son factores de incidencia permanente en tales interrelaciones; la meta siempre estará establecida en alcanzar la autorrealización de los implicados en el acto educativo.

\section{Avanzando un Cierre Reflexivo}

En términos Popperianos (1986) pudiéramos decir que se han producido cambios en el proceso educativo, resultado de innovaciones tecnológicas y, por tanto, en el mundo de los productos mentales (creación de la Internet, leyes, decretos, normativas, diseños curriculares), en el mundo físico institucional (desarrollo de plataformas virtuales de aprendizaje, diseño del aula virtual por el facilitador), pero no en los estados mentales de los actores, estudiantes y docentes (motivación, actitud y responsabilidad compartida ante la herramienta tecnológica). 
Por ello, el estudiante aún no se hace responsable por su propio aprendizaje como debe ser en la educación en línea, modalidad en la cual es una condición indispensable la puesta en práctica de las habilidades cognitivas de pensamiento y apertura mental que se posean (Sanz de Acedo, 2010) para gestionar el conocimiento de modo individual y colectivo, en colaboración con sus pares académicos. La reciente declaración a un diario digital de la región de un grupo de estudiantes rechazando el uso de la modalidad virtual para garantizar la prosecución de los estudios, instrucción emanada del Ministerio del Poder Popular para la Educación Universitaria Venezolano y como tal asumida por la IEUGP (https://www.el-carabobeno.com/estudiantes-de-laujap-exigen-aplazar-inscripcion-y-rechazan-clases-100-virtuales/), pone de manifiesto el camino aún por recorrer en cuanto al uso didáctico de las TIC, ya que los estudiantes en su declaración manifestaban "su rechazo al cronograma de inscripción impuesto por las autoridades y el plan de estudio a distancia, el cual propone que los estudiantes cursen su nuevo semestre a través de la plataforma Acrópolis 100\% virtual." (17-03-2020) (Resaltado nuestro).

Pero tampoco se observa en los docentes un cambio actitudinal favorable hacia el uso didáctico de las TIC, por cuanto no se evidencia el entusiasmo, la motivación para hacer de su docencia una actividad motivadora, creativa y diversa; en consonancia con la vigente Sociedad de la Información y del Conocimiento, sino la tendencia es a repetir sus patrones de enseñanza y permanecer en su zona de confort. Ya lo indicaba una docente formadora en TIC:

Los que en algún momento se atrevieron a hacerlo, lo hicieron "por cumplir un requisito de la cátedra" y "por salir de eso". No lo hicieron de manera amplia, sino que hicieron de sus espacios de trabajo repositorios de tareas y materiales digitalizados. Otros (docentes) [...] quizás por no dejar de hacer lo que han venido haciendo desde hace tiempo. (Conversación docente-autor, 2020)

Es notorio que las IEU venezolanas han reajustado sus estructuras y políticas internas en aras de garantizar la inserción efectiva y eficiente de las TIC en sus labores de docencia, investigación, extensión y gerencia. Nos referimos a su capacidad autopoiética (Maturana y Varela, 2003) que las obliga a reinventarse, transformarse y evolucionar. Pero, tal y como lo indicamos previamente, si los estados mentales de sus principales actores no están en sintonía o correspondencia con estos procesos de evolución y transformación, todo queda subyugado a una aspiración de las IEU y no se corresponde a las expectativas que todos sus actores deberían poseer, en pro del bien común, el progreso y el bienestar social. 
Los beneficios que se pueden obtener a través del uso eficiente y eficaz de las TIC en cualquier ámbito de la sociedad, es una realidad de constante y permanente presencia. A modo ilustrativo tenemos que el presente artículo fue totalmente estructurado superando las barreras geográficas de localización física de sus autores, gracias a una herramienta informática denominada Google Drive, parte de la suite gratuita que proporciona Google. Aunado a lo anterior, una situación sobrevenida a nivel mundial, tal y como lo constituye una pandemia de vertiginosa propagación, en la cual la reducción de la movilidad social es factor vital para frenar esa propagación; constituye una demostración fehaciente de cómo la diversidad de herramientas y recursos que ofrecen las TIC ha sido tomada como alternativa de Estado para proseguir las actividades de formación académica formal en distintos niveles y modalidades del sistema educativo, como es el caso de Venezuela y algunos estados de Estados Unidos de Norteamérica.

Esta experiencia nos anima a continuar indagando sobre las bondades didácticas de las TIC, cumpliendo así con el componente investigativo, en alianzas estratégicas interinstitucionales en Educación Universitaria, con lo cual ninguna Universidad se quedaría atrás y contribuiríamos con las acciones aceleradoras a ser desplegadas para alcanzar los Objetivos del Desarrollo Sostenible (2015), por cuanto, como bien se ha expresado, la educación es el sustrato viabilizador de los diecisiete objetivos formulados.

\section{Referencias}

Arreaza, E; Páez, H y De Sousa, L. (2013). Educar en valores mediante sistemas de gestión de aprendizaje. Ponencia presentada en la Novena Expedición Eduweb 2013: TIC, Educación y Formación. Universidad de Carabobo, Valencia, Venezuela.

BBC (18/10/2018) Carne in vitro: la empresa que produce pollo sin matar un solo animal. Recuperado de: https://www.bbc.com/mundo/noticias-45897953

Curci La Rocca, R. (2005). Diagnóstico de la Educación Superior virtual en Venezuela. Caracas: IESALC- UNESCO, IPASME. Recuperado de: www.iesalc.unesco.org.ve

Constitución de la República Bolivariana de Venezuela (1999). Gaceta Oficial de la República Bolivariana de Venezuela, 36.860, diciembre 30, 1999.

Decreto 3390. Publicado en Gaceta Oficial del 23 de diciembre del 2004, Número 336.626. Caracas. Venezuela. Recuperado de: http://www.tic.siteal.iipe.unesco.org/normativa/1207/decreto-ndeg825-may2000-acceso-yel-uso-de-internet-como-politica-prioritaria-para-el

El Carabobeño. (17-03-2020). Estudiantes de la UJAP exigen aplazar inscripción y rechazan clases $100 \%$ virtuales. Recuperado de: https://www.el-carabobeno.com/estudiantes-de-laujap-exigen-aplazar-inscripcion-y-rechazan-clases-100-virtuales/ 
El Economista.es. (07/02/2017). Cuero cultivado desde células: la alternativa real para quienes no quieren usar pieles de animal. Recuperado de: https://www.eleconomista.es/economia/noticias/8136561/02/17/El-cuero-biotecnologicola-alternativa-real-para-quienes-no-quieren-usar-pieles-de-animal.html

Facundo, A. (2005). Tecnologías de Información y Comunicación y Educación Superior Virtual en América Latina y el Caribe: Evolución, Características y Perspectivas. Bogotá: Los Libertadores/IESALC.

Goncalves, N. (2009). ¿Es posible la Integración Curricular de las TIC en la Educación Superior Venezolana? Una Experiencia con el Proyecto Génesis. Ponencia presentada en la Sexta Expedición Eduweb 2009. “TIC, Educación e Inclusión”. Universidad de Carabobo. Valencia, Venezuela.

Goncalves, N. (2011). La Plataforma Virtual Moodle y los Edublogs: Una Combinación para la Educación Superior. Ponencia presentada en la VII Jornadas Nacionales de Investigación Humanística y Educativa: Visiones para un Pensamiento en Libertad. Universidad Católica del Táchira. San Cristóbal, Venezuela.

Goncalves, N. (2015). Principios para un Modelo Integrador de las Tecnologías de Información y Comunicación al Currículo de la Educación Universitaria Venezolana. Tesis Doctoral. Universidad Nacional Experimental de las Fuerzas Armadas. Caracas, Venezuela.

Goncalves, N. (2018). Educación Universitaria Conectivista en Tiempos Inciertos. Revista Ciencias de la Educación. Volumen 28 No 51, enero-junio 2018, pp 306-325.

Joyanes, L. (1997). La Cibersociedad. Los Retos Sociales ante un Nuevo Mundo Digital. Madrid:McGraw-Hill Interamericana de España S.A.U.

Ley Orgánica de Educación (2009). Publicada en Gaceta Oficial № 5.929, del 15 de agosto del 2009.

Marín D., V. y Cabero A., J. (2010). Del conocimiento del estudiante universitario sobre las herramientas 2.0. Anales. Vol. 10, No 2 (Nueva Serie), 2010: 51-74 [archivo PDF]. Recuperado de: https://www.researchgate.net/publication/233926344_Del_conocimiento_del_estudiante_u niversitario_sobre_las_herramientas_20

Maturana, H. y Varela, F. (2003). De máquinas y seres vivos. Autopoiésis: la organización de lo vivo. 6ta ed. Buenos Aires: LUMEN.

Ministerio del Poder Popular para la Educación Superior (2009). Propuesta de Normativa para la Educación Superior a Distancia. Recuperado de: http://www.vracad.usb.ve/sites/default/files/EaD/Proyecto\%20Nacional\%20de\%20ES\%20 a\%20distancia.pdf

Ministerio del Poder Popular para la Educación Universitaria (2012). Propuesta de Normativa para la Educación Universitaria a Distancia. Recuperado de: http://ead.opsu.gob.ve/

Nagata, K. (2020). The Japan Times. It's a car. It's a plane. No, it's NEC's personal quadcopter (05-08-2019). Recuperado de: https://www.japantimes.co.jp/news/2019/08/05/business/car-plane-no-necs-personalquadcopter/\#.XUovw1X7RGE 
Organización de Naciones Unidas (2015). Objetivos del Desarrollo Sostenible. 25 de septiembre. Recuperado de https://www.un.org/sustainabledevelopment/es/objetivos-dedesarrollo-sostenible/

Páez, H. (2008). Alfabetización digital para docentes de postgrado. Revista Paradigma. [on line]. dic. 2008, vol.29, no.2. p.7-34. Recuperado de: http://www.scielo.org.ve/scielo.php?script=sci_arttext\&pid=S1011$22512008000200002 \& \operatorname{lng}=$ es\&nrm=iso

Páez, H. y Arreaza, E. (2013). Criterios para valorar la actuación competente del docente mediador en la era digital. Revista de Tecnología de Información y Comunicación en Educación. Volumen 7 No 2, julio-diciembre 2013, pp 23-36.

Popper, K. (1986). El Universo Abierto. Madrid. España: Editorial Tecnos.

Prensky, M (2001) Digital Natives, Digital Immigrants. NCB University Press, Vol. 9 No. 5, October.

Plan Nacional de Telecomunicaciones 2000-2011. Hacia la sociedad del conocimiento. Documento en línea. Recuperado de: http://lae.princeton.edu/catalog/009nj\#?c=0\&m=0\&s=0\&cv=0\&z=$0.3003 \% 2 \mathrm{C} 0.4491 \% 2 \mathrm{C} 1.7469 \% 2 \mathrm{C} 2.0617$

Presidencia de la República Bolivariana de Venezuela (2000). Decreto 825. Gaceta Oficial No. 36.955 de fecha 22 de mayo. Recuperado de: http://www.conatel.gob.ve/wpcontent/uploads/2017/01/sobre-internet.pdf

Proyecto Génesis (2009). Informe Curso Introductorio 2008-2009. Programa de Formación de Recursos Adicionales bajo la Modalidad Semipresencial y Virtual. Facultad de Ciencias de la Educación, Universidad de Carabobo.

Real Academia Española. Diccionario de la lengua española. Edición del tricentenario. Recuperado de: https://dle.rae.es/expectativa? $\mathrm{m}=$ form

Real Academia Española. Diccionario de la lengua española. Edición del tricentenario. Recuperado de: https://dle.rae.es/deber?m=form

Sanz de Acedo, M.L. (2010). Competencias cognitivas para la educación superior. Madrid: NARCEA, S.A. de Ediciones.

Siemens, G. (2004). A Learning Theory for the Digital Age. Elearnspace. Everything elearning. Recuperado de: http://www.elearnspace.org/Articles/connectivism.htm

UNESCO (2006). Informe sobre la Educación Superior en América Latina y el Caribe. 2000 2005. La Metamorfosis de la Educación Superior. Instituto Internacional de la UNESCO para la Educación Superior en América Latina y el Caribe (IESALC).

Universidad de Carabobo (2017). Undécima Expedición EDUWEB. TIC y Políticas Públicas en Educación. Su incidencia en el aula de clase. Facultad de Ciencias de la Educación, Bárbula, 18 al 20 de octubre.

Universidad José Antonio Páez (2018). Normas que Regulan el Uso de las Estrategias Virtuales como Apoyo a la Presencialidad. Consejo Universitario No. 535 de fecha 8 de agosto de 2018. 